\title{
The Relationship Between Intuitive Eating and Diet Quality in a College Population
}

\author{
Marta Tabatabai, Julie Holland, Laura Curtis $\mathbb{0}$, \\ and Michelle Neyman Morris (i) \\ California State University Chico, Department of Nutrition \& Food Science
}

\begin{abstract}
Background and Purpose: Many college students have nutrient poor and energy dense diets and are also more likely to experience poor body image, which can result in unsafe dieting behaviors for the purpose of managing weight. Intuitive eating is an alternative approach to dieting that focuses on physiological hunger and fullness cues, while eating for both satisfaction and health without restriction of any foods. Methods: This study examined the association between intuitive eating and diet quality in a college population. College students, aged 18-56 years, completed an online survey which assessed intuitive eating using the Intuitive Eating Scale-2 (IES-2) and diet quality using the Starting The Conversation (STC) simplified food frequency instrument. Results: IES-2 total score was positively correlated with higher overall diet quality and was negatively correlated with fast food and chip consumption. Eating for physical rather than emotional reasons and body-food choice congruence IES-2 subscales were positively correlated with diet quality while the unconditional permission to eat subscale was negatively correlated with diet quality. Conclusion: Strategies that focus on eating for health and well-being and minimize emotional eating are associated with higher overall diet quality and may be incorporated in dietary interventions among college students aimed at promoting healthy behaviors.
\end{abstract}

(C) 2021 and CC-BY 4.0 licensed by the authors.

Keywords: diet, college students, intuitive eating

\section{Introduction}

Healthy dietary patterns are particularly important today with over half of Americans having at least one chronic disease related to poor diet (Dietary Guidelines, 2020). The World Health Organization defines a healthy diet as one rich in fruits, vegetables, legumes, nuts and whole grains, as well as low in salt, free sugars, and saturated fat (Healthy Diet, 2018). A majority of Americans are not eating adequate amounts of fruits and vegetables and are exceeding recommended intakes of added sugar, salt, and saturated fat (Dietary Guidelines, 2020). College students are no exception. They tend to lack adequate fruit, vegetable, and whole grain intake and over consume less nutrient-dense options such as cookies, chips, and ice cream (Downes, 2015; Strong, Parks, Anderson, Winett, \& Davy, 2008). As college students transition to having greater autonomy over their food choices, they appear to be choosing fewer nutritious options.

Recent attention has also focused on food insecurity among college students. Food insecurity refers to having unreliable access to adequate quantities of food due to insufficient finances or resources (United States Department of Agriculture, 2019). Stress and poor nutrition due to food insecurity can increase risk of chronic 
disease (Seligman \& Shillinger, 2010). Research has shown that $11 \%$ of U.S households and anywhere from $21 \%$ to $52 \%$ of students struggle with food insecurity (United States Department of Agriculture, 2019; Crutchfield \& Maguire, 2018). Due to limited resources, food insecure college students may eat less or alternatively, they may opt for more affordable calorically dense yet nutrient poor foods, thereby negatively impacting their overall diet quality.

In addition to poor eating habits, college students are exposed to many new stressors during this life transition which may lead to unhealthy behaviors and can increase the risk for poor body image. Many young adults, especially those who have changes in their body mass index (BMI) over time, tend to be less satisfied with their bodies, perceive themselves to be fatter than ideal, and report their ideal body size to be smaller (Mintem, Gigante, \& Horta, 2015; Paap \& Gardner, 2011). Poor body image often results in dieting and disordered eating habits (Haynos, Watts, Loth, Pearson, \& Neumark-Stzainer, 2016). In adolescence, dieting, or restricting calories or food quantities in an effort to influence body weight, has been shown to be the most significant precursor to developing an eating disorder (Patton, Selzer, Coffey, Carlin, \& Wolfe, 1999). Furthermore, dieting and other disordered eating behaviors are prevalent among college students (Lipson \& Sonneville, 2017). Dieting may lead people to distrust their bodies' physiological cues of hunger and fullness and to become preoccupied with food, negatively impacting their relationship with food (Tribole \& Resch, 2020). In addition, over time dieting is correlated with binge eating, greater weight gain, low mood, loss of bone and lean mass, compromised immunity and skeletal integrity, poor body image, and weight cycling (Bombak, Monaghan, $\&$ Rich 2019). Weight cycling as a result of yoyo dieting can lead to worse metabolic health. (Montani, Schutz, \& Dulloo, 2015). It is for these reasons that dieting is viewed as being detrimental to both physiological and psychological well-being.
Intuitive eating (IE) practices are in sharp contrast to typical dieting behaviors. IE is based on the premise that individuals are the experts of their own bodies (Tribole \& Resch, 2020). It promotes interoceptive awareness, the ability to tune into and honor internal cues of hunger and fullness, while eating for both satisfaction and nourishment without dietary restraint. Upon the rejection of the diet mentality, IE encourages practicing gentle nutrition while eating for health, well-being, and enjoyment (Tribole \& Resch, 2020). In previous studies, IE has been associated with lower levels of disordered eating and a more positive body image (Bruce \& Ricciardelli, 2016; Linardon \& Mitchell, 2017). Interventions using IE have led to decreased depression and anxiety, increased self-esteem, and improved blood pressure, blood lipid levels, and cardiorespiratory fitness among participants (Schaefer \& Magnuson, 2014). Thus, the shift away from dieting towards IE can enhance both physical and mental well-being.

For some people, a barrier to adopting IE and giving themselves permission to eat all foods may be the fear that they will overeat unhealthy foods and gain weight. However, the limited literature on this topic suggests that IE is correlated with the consumption of a healthier diet. IE has been associated with a lower BMI and with decreased intake of ultra-processed, high fat, and high sugar foods (Carbonneau et al., 2017; Ulian et al., 2018; Van Dyke \& Drinkwater, 2013). Small positive associations of diet quality with two IE subscales were observed in women (Horwath, Hagmann, \& Hartmann, 2019). Eating based on internal cues has also been associated with higher intakes of fruits, vegetables, and whole grains. (Lohse, Bailey, Krall, Wall, \& Mitchell, 2011). Additionally, IE and diet quality both improved during a Health at Every Size ${ }^{\circledR}$ (HAES ${ }^{\circledR}$ ) intervention (Carbonneau et al., 2017).

\section{The Current Study}

Few studies have examined the relationship between IE and diet quality and to our knowledge, it has not yet been explored in a 
college population. Since it is known that college students eat less nutrient dense diets and are at risk of dieting and disordered eating behaviors, it is important to evaluate this relationship. The aim of this study was to examine the relationship between IE and its four subscales, unconditional permission to eat, eating for physical rather than emotional reasons, reliance on hunger and satiety cues, and body-food choice congruence, with diet quality in a college population.

\section{Methods}

\section{Study Design and Participants}

This was a cross sectional study among college students enrolled at a midsize western university in the spring semester of 2020. Based on a sample size calculation using G-power a total of 459 participants were needed $(a=0.05,1-b=0.8)$. Students were recruited via email invitation, class announcements, and social media to complete an online survey. Inclusion criteria included: 18 years or older, undergraduate or graduate student, and currently enrolled in classes. As an incentive for completing the survey, students were entered into a raffle to win one of two $\$ 25$ gift cards. All procedures were approved by the University Human Subjects in Research Committee.

\section{Measures and Procedures}

An online survey created through Qualtrics was used to collect data on demographics, intuitive eating, and diet quality. Demographics collected included age, gender, major, race/ethnicity, year in school, Pell Grant recipient status (to assess socioeconomic status and potentially food insecurity), nutrition course completion, selfreported height, weight, and days of physical activity per week.

The previously validated Intuitive Eating Scale-2 was used to assess intuitive eating status, the independent variable (Tylka \& Kroon Van Diest, 2013). The scale has 23 questions with four subgroups including unconditional permission to eat, eating for physical rather than emotional reasons, reliance on hunger and satiety cues, and body-food choice congruence. Responses were on a 5-point Likert scale ranging from "strongly disagree" to "strongly agree." The scores were determined by adding together all items and dividing by the total number of questions or number of questions in each subgroup to create an average score. The scale resulted in a total score as well as scores for each of the subgroups. Final scores for each ranged from 1 to 5 with a higher score being more representative of an intuitive eater.

The previously validated Starting The Conversation (STC) simplified food frequency instrument was used to assess diet quality, the dependent variable (Paxton, Strycker, Toobert, Ammerman, \& Glasgow, 2011). This survey was validated in an adult population and chosen for this study because it is brief and easy to use, therefore likely resulting in a higher response rate for online survey administration. The STC consisted of eight questions about consumption of fast food, fruits, vegetables, sodas, lean proteins, chips, desserts, and fat over the past month with three response options for each question. The left column was the most healthful dietary option (scored 0 ), the middle column was less healthful (scored 1), and the right column was the least healthful (scored 2). A diet summary score was calculated with 16 being the highest and 0 being the lowest possible. A lower score was indicative of higher diet quality.

\section{Analyses}

Data were analyzed using Statistical Package for the Social Sciences (SPSS) (IBM Corp. Released 2017. IBM SPSS Statistics for Macintosh, Version 25.0. Armonk, NY: IBM Corp.) A pvalue of $<0.05$ was used to assess significance. One way ANOVA and Chi Square tests were used to assess demographics so as to examine factors associated with intuitive eating and diet quality. Exploratory analyses were used to reduce the number of potential confounders. Any potential confounder that had at least a modest $(p<0.100)$ relation with diet quality or intuitive eating was included in later multivariate models. 
Multiple linear regression was conducted to assess the association between the STC for all four subscales of the IES-2 and the total IES-2 score while controlling for age, ethnicity, class standing, nutrition class completion, and physical activity. The relationship between IES- 2 and each of the eight food items was also analyzed using multiple linear regression while controlling for covariates.

\section{Results}

After providing informed consent, 312 college students completed the survey. Five individuals were excluded from analysis due to not selecting a gender. The final sample of 307 students was $75.2 \%$ female with a mean age of 23.5 years (SD $=6.6$, range $=18-56)$, and mean BMI of 24.5 (SD $=5.0$, range $=15.3-54.9)$ (Table 1). Over half $(56.4 \%)$ of participants were juniors and seniors, and $35.9 \%$ were Pell Grant eligible. The mean physical activity level was 3.6 days a week $(\mathrm{SD}=1.9$, range $=0-7)$. Nearly half of participants identified as White $(48.3 \%)$, while $19.5 \%$ identified as Hispanic and $19.5 \%$ identified as Asian.

The following factors had a modest association with intuitive eating and were included in the model: age, ethnicity, class standing, nutrition class completion, and physical activity. BMI and gender were not associated with the variables. Associations between intuitive eating and diet quality were examined. There was an inverse correlation between Intuitive Eating Scale-2 (IES-2) total score and Starting the Conversation (STC) summary score after controlling for covariates, indicating that intuitive eaters (those who scored high on IES-2) had significantly better diet quality $(p=.029)$ (Table 2). Eating for physical rather than emotional reasons and bodyfood choice congruence IES-2 subscales were also inversely correlated with STC summary score $(\mathrm{p}=.013, \mathrm{p}=.000)$.
Table 1.

Sociodemographic Characteristics of the Study Population $(\mathrm{N}=307)$

\begin{tabular}{lr}
\hline & $\begin{array}{r}\text { Mean } \\
\text { (SD) or } \%\end{array}$ \\
Gender & \\
Male & $23.8 \%$ \\
Female & $75.2 \%$ \\
Transgender & $1.0 \%$ \\
Age, years & $23.5 \pm 6.6$ \\
BMI (kg/m ${ }^{2}$ ) & $24.5 \pm 5.0$ \\
Health Majors ${ }^{1}$ & $39.1 \%$ \\
Physical Activity, days/ week & $3.6 \pm 1.9$ \\
Class standing & \\
Freshman & $11.5 \%$ \\
Sophomore & $14.7 \%$ \\
Junior & $27.2 \%$ \\
Senior & $29.2 \%$ \\
Graduate & $17.3 \%$ \\
Pell Grant & $35.9 \%$ \\
Taken a Nutrition Class & $61.5 \%$ \\
Ethnicity/Race & \\
African American & $1.3 \%$ \\
Asian & $19.5 \%$ \\
Hispanic & $19.5 \%$ \\
Native Hawaiian & $0.3 \%$ \\
2+ ethnicities/races & $10.9 \%$ \\
White & $48.3 \%$ \\
\hline Note. Reported as mean \pm SD or $\%$ & \\
Kinesiology, Exercise Physiology, Pre-Nursing, \\
Nutrition, Public Health, Nursing, Physical \\
Education & \\
& \\
\hline
\end{tabular}

The unconditional permission to eat subscale was the only IES-2 subscale that was positively correlated with STC summary score, indicating poorer diet quality $(p=.001)$. The reliance on hunger and satiety cues subscale was not significantly related to diet quality. With regards to the covariates, health major, physical activity, and class standing were inversely correlated with STC summary scores in all models. 
Table 2.

Linear regression on Starting The Conversation (STC) Summary Score with Intuitive Eating Scale-2 (IES-2) Total Score and the Four IES-2 Subscales ( $\mathrm{N}=307)$

\begin{tabular}{|c|c|c|c|c|c|}
\hline & Model 1 & Model 2 & Model 3 & Model 4 & Model 5 \\
\hline IES-2 Total Score & $-0.543^{*}$ & - & - & - & - \\
\hline $\begin{array}{l}\text { Unconditional Permission to Eat } \\
\text { Eating for Physical (not Emotional) }\end{array}$ & - & $0.598 * *$ & - & - & - \\
\hline Reasons & - & - & $-0.411 *$ & - & - \\
\hline Reliance on Hunger and Satiety Cues & - & - & - & -.0271 & - \\
\hline Body-Food Choice Congruence & - & - & - & - & $-1.287 * *$ \\
\hline Taken a Nutrition Class & -0.098 & -0.179 & -0.147 & -0.090 & 0.125 \\
\hline \multicolumn{6}{|l|}{ Ethnicity/Race } \\
\hline Asian & 0.367 & 0.380 & 0.346 & 0.386 & 0.523 \\
\hline Hispanic & 0.578 & 0.477 & 0.563 & 0.559 & 0.439 \\
\hline Other $^{1}$ & -0.554 & -0.578 & -0.548 & -0.583 & -0.543 \\
\hline Health Majors ${ }^{2}$ & $-0.836^{* *}$ & $-0.968 * *$ & $-0.832 * *$ & $-0.886^{* *}$ & $-0.730 * *$ \\
\hline Age & -0.016 & -0.017 & -0.015 & -0.018 & -0.008 \\
\hline Physical Activity & $-0.292 * *$ & $-0.285^{* *}$ & $-0.288 * *$ & $-0.301 * *$ & $-0.165^{*}$ \\
\hline Class standing $^{3}$ & $-0.468^{* *}$ & $-0.539 * *$ & $-0.492 * *$ & $-0.475 * *$ & $-0.440 * *$ \\
\hline
\end{tabular}

Note. $* \mathrm{p} \leq .05, * * \mathrm{p} \leq 0.01$, white is the reference group, IES-2 = Intuitive Eating Scale- 2

${ }^{1}$ African American, Native Hawaiian/Pacific Islander, Two or more ethnicities

${ }^{2}$ Kinesiology, Exercise Physiology, Pre-Nursing, Nutrition, Public Health, Nursing, Physical Education

${ }^{3}$ Freshman, sophomore, junior, senior, graduate student

The relationship between intuitive eating and diet quality was further investigated by examining individual food items included in the STC survey. IES-2 scores were negatively correlated with fast food and chip consumption, after controlling for covariates $(p=.008, p=.044)$ (Table 3). However, fruit, vegetable, soda, lean protein, dessert, and fat consumption were not significantly correlated with IES-2 scores. Choosing a health major was inversely correlated with fast food and soda consumption $(\mathrm{p}=.033, \mathrm{p}=.001)$, while physical activity was inversely correlated with chip consumption $(p=.004)$. Fruit, vegetable, and lean protein intake was positively correlated with physical activity levels $(\mathrm{p}=.001, \mathrm{p}=.003, \mathrm{p}=.004)$. In addition, higher class standing was positively correlated with vegetable consumption $(\mathrm{p}=.005)$ and negatively correlated with fast food, soda, and chip consumption $(\mathrm{p}=.037, \mathrm{p}=.009, \mathrm{p}=.000)$. Asian race was positively correlated with soda consumption and negatively correlated with lean protein consumption. Lastly, Hispanic ethnicity was negatively correlated with vegetable consumption.

\section{Discussion}

This study demonstrated an inverse relationship between IES-2 total score and STC diet summary score after controlling for age, ethnicity, class standing, nutrition class completion, and physical activity. This indicates that those who eat more intuitively have a more healthful diet. 
Table 3.

Linear Regression on Starting The Conversation (STC) food items with Intuitive Eating Scale-2 (IES2) total score $(\mathrm{N}=307)$

\begin{tabular}{lcccccccc}
\hline & $\begin{array}{c}\text { Fast } \\
\text { food }\end{array}$ & Fruit & Vegetables & Sodas $^{1}$ & $\begin{array}{c}\text { Lean } \\
\text { Proteins }^{2}\end{array}$ & Chips $^{3}$ & Desserts & Fat $^{4}$ \\
\hline $\begin{array}{l}\text { IES-2 Total } \\
\text { Taken }\end{array}$ & $-0.200^{* *}$ & 0.093 & -0.010 & -0.056 & 0.010 & $-0.147^{*}$ & -0.063 & 0.018 \\
$\begin{array}{l}\text { Nutrition Class } \\
\text { Ethnicity/Race }\end{array}$ & 0.073 & 0.007 & 0.118 & 0.115 & -0.018 & -0.090 & 0.041 & -0.131 \\
$\quad$ & & & & & & & & \\
$\quad$ Asian & 0.071 & 0.009 & -0.085 & $0.230^{* *}$ & $-0.247^{*}$ & -0.153 & -0.200 & 0.097 \\
$\quad$ Hispanic & 0.166 & -0.056 & $-0.266^{* *}$ & 0.047 & 0.017 & 0.115 & -0.075 & 0.021 \\
$\quad$ Other $^{5}$ & -0.058 & -0.048 & -0.004 & -0.075 & 0.057 & -0.217 & -0.204 & 0.005 \\
Health Majors & & & & & & & \\
Age & $-0.184^{*}$ & 0.011 & 0.063 & $-0.209^{* *}$ & 0.061 & -0.058 & -0.174 & -0.076 \\
Physical & -0.004 & 0.005 & 0.000 & -0.002 & 0.011 & 0.001 & 0.006 & -0.001 \\
Activity & -0.040 & $0.056^{* *}$ & $0.062^{* *}$ & -0.020 & $0.058^{* *}$ & $-0.047^{*}$ & -0.008 & -0.002 \\
Class standing & $-0.070^{*}$ & -0.012 & $0.094^{* *}$ & $-0.061^{* *}$ & 0.030 & $-0.130^{* *}$ & 0.056 & -0.037 \\
& & & & & & & & \\
\hline
\end{tabular}

Note. ${ }^{*} \mathrm{p} \leq .05,{ }^{*} \mathrm{p} \leq 0.01$, white is the reference group, IES-2 = Intuitive Eating Scale-2

${ }^{1}$ Sodas was composed of sodas and sweet tea.

${ }^{2}$ Lean proteins was composed of beans, chicken, and fish.

${ }^{3}$ Chips was composed of chips or crackers (not low-fat).

${ }^{4}$ Fat was composed of margarine, butter, or meat fat.

${ }^{5}$ African American, Native Hawaiian/Pacific Islander, Two or more ethnicities

${ }^{6}$ Kinesiology, Exercise Physiology, Pre-Nursing, Nutrition, Public Health, Nursing, Physical Education ${ }^{7}$ Freshman, sophomore, junior, senior, graduate student

These results are similar to previous studies, where eating based on internal cues was associated with higher intakes of fruits, vegetables, and whole grains, suggesting a more nutritious diet (Lohse, Bailey, Krall, Wall, \& Mitchell, 2011). In addition, a randomized controlled trial comparing a weight-neutral approach with a weight-loss approach found the weight-neutral group had a significant increase in intuitive eating along with fruit and vegetable intake. (Mensinger, Calogero, Stranges, \& Tylka, 2016). Another weight-neutral intervention based on the principles of Health at Every Size $\mathbb{R}$ (HAES ${ }^{\circledR}$ ) was effective in improving intuitive eating and diet quality, however, the positive relationship between the two was unclear outside of the HAES ${ }^{\circledR}$ intervention (Carbonneau et al., 2017).
The eating for physical rather than emotional reasons IES-2 subscale reflects emotional eating, while the body-food choice congruence subscale relates to eating nutritious foods that support body function and health. In this study both of these were inversely correlated with the STC diet summary score, indicating higher diet quality as subscale scores increased. This is consistent with findings from a large cross sectional study that found higher physical reasons and cues scores were associated with lower sweet and fatty food intake in both genders (Camalleri et al., 2016). In addition, small positive associations of diet quality with eating for physical rather than emotional reasons were observed in women (Horwath, Hagmann, \& Hartmann, 2019). As hypothesized, we observed that the tendency to honor gentle nutrition, a principle of intuitive 
eating, by choosing foods that offer energy and nutrition was related to diet quality. To our knowledge only one other study has examined the relationship between the IES-2 body-food choice congruence subscale and diet quality, however, no association was found between the two (Horwath, Hagmann, \& Hartmann, 2019).

In contrast, the unconditional permission to eat subscale was positively correlated with the STC diet summary score, indicating a less healthful diet as study participants give themselves greater unconditional permission to eat. The permission subscale was used to assess if certain foods are avoided and if dieting rules are in place. The current findings are in line with previous studies that found high scores for unconditional permission to eat were associated with poorer diet quality (Horwath, Hagmann, \& Hartmann, 2019, Camalleri et al., 2016). Greater consumption of sweets, fatty foods, fast food, pizza, and quiches and less fruit, vegetable, and whole grain intake were observed in individuals with high permission to eat scores (Camalleri et al., 2016). It appears that those who have more restrictions around food tend to eat more nutrient dense foods and limit foods high in salt, sugar, and fat, at least in the short term. It is important to note that continuous restriction often leads to binge eating and intuitive eating has been negatively associated with binge eating (Madden, Leong, Gray, \& Horwath, 2012). While unconditional permission to eat may not have a positive impact on diet quality on its own, when combined with the other components of intuitive eating that focus on limiting emotional eating and eating for health, overall diet quality is enhanced.

Total intuitive eating scores were negatively associated with fast food, chip, and cracker consumption. Interestingly, no relationships were seen with other food items, including fruit and vegetable consumption, although the body-food choice congruence subscale was associated with diet quality. A previous cross sectional study in a college population also observed no association between total intuitive eating score and fruit and vegetable intake (Barad et al., 2019). Another study found no relationship between intuitive eating and fruit, vegetable, dessert, burger, chip, meat pie, and sausage roll intake (Madden, Leong, Gray, \& Horwath, 2012). However, the results from the Madden study are not directly comparable to our findings because the survey was conducted in a population of middle-age women in New Zealand.

Given that a key aspect of intuitive eating is making food choices that honor health and support the feeling of wellness, it was hypothesized that intuitive eating would be positively associated with diet quality. Intuitive eaters may instinctively know that frequent consumption of fast foods, chips, and crackers leaves them feeling unwell. A participant in a qualitative intuitive eating study stated that "if you're listening to your body, what your body wants is something that meets that physical need" (Barraclough, Hay-Smith, Boucher, Tylka, \& Horwath, 2019). Increased nutrition knowledge and 'body wisdom' (i.e., following body signals to guide food choices) are congruent with intuitive eating. The current findings suggest that those whose eating behaviors are consistent with intuitive eating principles are no more likely to have poor diets.

Secondary analysis showed an inverse association between health majors, physical activity level, class standing, and STC summary score. As hypothesized, those majoring in programs with a health and wellness focus consumed a more healthful diet. Positive changes in eating behaviors of college students over time were also seen in a previous study, where $40.5 \%$ of college students reported eating more vegetables and $38.3 \%$ reported eating more fruits since enrolling in college (Hilger, Loerbroks, Diehl, 2016).

\section{Limitations}

The cross sectional study design prevented the ability to determine a causal relationship. Additionally, the final sample size was a convenience sample and smaller than the G- 
power calculation. The sample was $75 \%$ female, which isn't representative of most college populations and limits the generalizability of these results. Next, food intake was self-reported through a simplified food frequency instrument. This questionnaire contains eight food items and only consists of three ranges of intake frequencies to choose from, which could have oversimplified diet quality. A precise analysis of energy and nutrient intake was not conducted in this study, nor was consideration given to other factors that may influence the diets of college students, such as alcohol and recreational drug use. Lastly, a general limitation of food frequency questionnaires is that individuals may over- or under-report their food intake. Despite having a small sample size, significant results were observed in this study and more research on the relationship between intuitive eating and diet quality is warranted. In addition, while there are pros and cons associated with all dietary intake assessments, the STC appears to be a feasible tool for a college population due to its simplicity and ease of use.

\section{Conclusion}

This study supports intuitive eating as a health promoting behavior among college students who may be at risk for poor diet quality, disordered eating, and food insecurity. Intuitive eating does not encourage cutting out or emphasizing certain foods, as typical diets do, but instead uses nutrition knowledge along with internal cues of hunger and fullness to determine what, how much, and when to eat. Strategies that focus on eating for health and well-being and minimize emotional eating are associated with higher overall diet quality and may be incorporated in dietary interventions among college students aimed at promoting healthy behaviors. Further research is needed to explore this association and any possible causal relationships in larger and more diverse populations.

\section{References}

Barad, A., Cartledge, A., Gemmill, K., Misner, N. M., Santiago, C. E., Yavelow, M., \& Langkamp-Henken, B. (2019). Associations between intuitive eating behaviors and fruit and vegetable intake among college students. Journal of Nutrition Education and Behavior, 51(6), 758-762. doi: 10.1016/j.jneb.2019.03.010

Barraclough, E. L., Hay-Smith, E. J. C., Boucher, S. E., Tylka, T. L., \& Horwath, C. C. (2019).Learning to eat intuitively: A qualitative exploration of the experience of mid-age women. Health Psychology Open, 6(1), 1-8. doi: 10.1177/2055102918824064

Bombak, A., Monaghan, L. F., \& Rich, E. (2018). Dietary approaches to weight-loss, Health At Every Size ${ }^{\circledR}$ and beyond: Rethinking the war on obesity. Social Theory \& Health, 17(1), 89-108. doi: 10.1057/s41285-018-0070-9

Bruce, L. J., \& Ricciardelli, L. A. (2016). A systematic review of the psychosocial correlates ofintuitive eating among adult women. Appetite, 96, 454-472. doi: 10.1016/j.appet.2015.10.012

Camilleri, G. M., Méjean, C., Bellisle, F., Andreeva, V. A., Kesse-Guyot, E., Hercberg, S., \& Péneau, S. (2016). Intuitive eating dimensions were differently associated with food intake in the general population-based NutriNet-Santé study. The Journal of Nutrition, 147(1), 61-69. doi: 10.3945/jn.116.234088

Carbonneau, E., Bégin, C., Lemieux, S., Mongeau, L., Paquette, M., Turcotte, M., \& Provencher, V. (2017). A Health at Every Size intervention improves intuitive eating and diet quality in Canadian women. Clinical Nutrition, 36(3), 747-754. doi: 10.1016/j.clnu.2016.06.008

Crutchfield, R. M. \& Maguire, J. (2018). Study of Student Basic Needs. California State University. website, https://www.calstate.edu/basicneeds 


\section{INTUITIVE EATING AND DIET QUALITY IN A COLLEGE POPULATION}

Downes, L. (2015). Physical activity and dietary habits of college students. The Journal for Nurse Practitioners, 11(2). doi: 10.1016/j.nurpra.2014.11.015

Food Security Status of U.S. Households in 2018. (2019). U.S. Department of Agriculture. Retrieved from https://www.ers.usda.gov/topics/food-nutrition-assistance/food-security-in-the-us/key-statisticsgraphics.aspx

Haynos, A. F., Watts, A. W., Loth, K. A., Pearson, C. M., \& Neumark-Stzainer, D. (2016). Factors predicting an escalation of restrictive eating during adolescence. Journal of Adolescent Health, 59, 391-396. doi: 10.1016/j.adohealth.2016.03.011

Healthy Diet. (2018, October 23). World Health Organization. Retrieved from https://www.who.int/newsroom/fact-sheets/detail/healthy-diet

Hilger, J., Loerbroks, A., \& Diehl, K. (2017). Eating behaviour of university students in Germany: dietary intake, barriers to healthy eating and changes in eating behaviour since the time of matriculation. Appetite, 109, 100-107. doi: 10.1016/j.appet. 2016.11.016

Horwath, C., Hagmann, D., \& Hartmann, C. (2019). Intuitive eating and food intake in men and women: Results from the Swiss food panel study. Appetite, 135, 61-71. doi: 10.1016/j.appet.2018.12.036

Linardon, J., \& Mitchell, S. (2017). Rigid dietary control, flexible dietary control, and intuitive eating: Evidence for their differential relationship to disordered eating and body image concerns. Eating Behaviors, 26, 16-22. doi: 10.1016/j.eatbeh.2017.01.008

Lipson, S. K., \& Sonneville, K. R. (2017). Eating disorder symptoms among undergraduate and graduate students. Eating Behaviors, 24, 81-88. doi: 10.1016/j.eatbeh.2016.12.003

Lohse, B., Bailey, R. L., Krall, J. S., Wall, D. E., \& Mitchell, D. C. (2012). Diet quality is related to eating competence in cross-sectional sample of low-income females surveyed in Pennsylvania. Appetite, 58(2), 645-650. doi: 10.1016/j.appet.2011.11.022

Madden, C. E., Leong, S. L., Gray, A., \& Horwath, C. C. (2012). Eating in response to hunger and satiety signals is related to BMI in a nationwide sample of 1601 mid-age New Zealand women. Public Health Nutrition, 15(12), 2272-2279. doi: 10.1017/s1368980012000882

Mensinger, J. L., Calogero, R. M., Stranges, S., \& Tylka, T. L. (2016). A weight-neutral versus weightloss approach for health promotion in women with high BMI: A randomized-controlled trial. Appetite, 105, 364-374. doi: 10.1016/j.appet.2016.06.006

Mintem, G. C., Gigante, D. P., \& Horta, B. L. (2015). Change in body weight and body image in young adults: A longitudinal study. BMC Public Health, 15(1). doi: 10.1186/s12889-015-1579-7

Montani, J.-P., Schutz, Y., \& Dulloo, A. G. (2015). Dieting and weight cycling as risk factors for cardiometabolic diseases: Who is really at risk? Obesity Reviews, 16(1), 7-18. doi: 10.1111/obr.12251

Paap, C. E., \& Gardner, R. M. (2011). Body image disturbance and relationship satisfaction among college students. Personality and Individual Differences, 51(6), 715-719. doi: 10.1016/j.paid.2011.06.019

Patton, G. C., Selzer, R., Coffey, C., Carlin, J. B., \& Wolfe, R. (1999). Onset of adolescent eating disorders: population based cohort survey over 3 years. BMJ Clinical Research, 318(7186). doi: 10.1136.bmj.318.7186.765

Paxton, A. E., Strycker, L. A., Toobert, D. J., Ammerman, A. S., \& Glasgow, R. E. (2011). Starting the conversation: performance of a brief dietary assessment and intervention tool for health professionals. American Journal of Preventive Medicine, 40(1). doi: 10.1016/s07493797(10)00687-2

Schaefer, J. T., \& Magnuson, A. B. (2014). A review of interventions that promote eating by internal cues. Journal of the Academy of Nutrition and Dietetics, 114(5), 734-760. doi: 10.1016/j.jand.2013.12.024 
INTUITIVE EATING AND DIET QUALITY IN A COLLEGE POPULATION

Seligman, H. K., \& Shillinger, D. (2010). Hunger and Socioeconomic Disparities in Chronic Disease. The New England Journal of Medicine, 363, 6-9. doi: 10.1056/NEJMp1000072

Strong, K. A., Parks, S. L., Anderson, E., Winett, R., \& Davy, B. M. (2008). Weight gain prevention: identifying theory-based targets for health behavior change in young adults. Journal of the American Dietetic Association, 108(10), 1708-1705. doi: 10.1016/j.jada.2008.07.007

Tribole, E., \& Resch, E. (2020). Intuitive Eating: A revolutionary anti-diet approach (4th ed.). St. Martin's Essentials. website, https:/www.overdrive.com/search?q=FF22AEFE-3400-44FEAF91-A844023C9AFB

Tylka, T. L., \& Diest, A. M. K. V. (2013). The Intuitive Eating Scale-2: Item refinement and psychometric evaluation with college women and men. Journal of Counseling Psychology, 60(1), 137-153. doi: 10.1037/a0030893

Ulian, M. D., Pinto, A. J., Sato, P. D., Benatti, F. B., Campos-Ferraz, P. L., Coelho, D., Scagliusi, F. B. (2018). Effects of a new intervention based on the Health at Every Size approach for the management of obesity: The "Health and Wellness in Obesity" study. Plos One, 13(7). doi: 10.1371/journal.pone.0198401

U.S. Department of Agriculture and U.S. Department of Health and Human Services. (2020, December) Dietary Guidelines for Americans, 2020-2025. https://www.dietaryguidelines.gov/

Van Dyke, N., \& Drinkwater, E. J. (2013). Relationships between intuitive eating and health

indicators: Literature review. Public Health Nutrition, 17(8), 1757-1766. doi: 10.1017/s1368980013002139

Corresponding Author Information

Michelle Neyman Morris, PhD, RD

Professor, Department of Nutrition \& Food Science

California State University, Chico

Chico, CA 95929-0002

530-898-4757

mrmorris@,csuchico.edu 